\title{
5/8 HLA Match
}

National Cancer Institute

\section{Source}

National Cancer Institute. 5/8 HLA Match. NCI Thesaurus. Code C158039.

Degree of HLA match is 5/8 alleles with fewer than 2 mismatches per locus at HLA-A, -B, $-C$ and -DRB1. 